\title{
Colovesical fistula causing an uncommon reason for failure of computed tomography colonography: a case report
}

Angeliki Neroladaki, Romain Breguet, Diomidis Botsikas, Sylvain Terraz, Christoph D Becker and Xavier Montet

\begin{abstract}
Introduction: Computed tomography colonography, or virtual colonoscopy, is a good alternative to optical colonoscopy. However, suboptimal patient preparation or colon distension may reduce the diagnostic accuracy of this imaging technique.

Case presentation: We report the case of an 83-year-old Caucasian woman who presented with a five-month history of pneumaturia and fecaluria and an acute episode of macrohematuria, leading to a high clinical suspicion of a colovesical fistula. The fistula was confirmed by standard contrast-enhanced computed tomography. Optical colonoscopy was performed to exclude the presence of an underlying colonic neoplasm. Since optical colonoscopy was incomplete, computed tomography colonography was performed, but also failed due to inadequate colon distension. The insufflated air directly accumulated within the bladder via the large fistula.
\end{abstract}

Conclusions: Clinicians should consider colovesical fistula as a potential reason for computed tomography colonography failure.

\section{Introduction}

Computed tomography colonography (CTC) or virtual colonoscopy has gained wide acceptance and is now considered as an excellent alternative in case of incomplete or contraindicated conventional optical colonoscopy (OC) [1]. However, suboptimal patient preparation or suboptimal colon distension may reduce the diagnostic accuracy of CTC.

Even though CTC is a well-recognized method for colon analysis and detection of lumen abnormalities, some pitfalls and limitations may prevent an adequate bowel analysis. A few other publications have reported colovesical fistula diagnosed with CTC, but with adequate distension of the colon [2,3]. To the best of our knowledge, we report the second case of inadequate colon distension due to an important colovesical fistula in the context of colonic diverticulitis [4].

\footnotetext{
* Correspondence: xavier.montet@hcuge.ch

* Correspondence: xavier.montet@hcuge.ch 4, 1211, Geneva 4, Switzerland
}

\section{Case presentation}

An 83-year-old Caucasian woman presented with a fivemonth history of pneumaturia and fecaluria and an acute episode of macrohematuria, leading to a high clinical suspicion of a colovesical fistula. Our patient also reported a weight loss of $5 \mathrm{~kg}$ in a short period of time but had no other significant clinical history. The initial contrast-enhanced computed tomography (CT) clearly depicted the suspected colovesical fistula (Figure 1).

An OC was performed to investigate the colon for the potential presence of a colic neoplasm associated with the colovesical fistula. The $\mathrm{OC}$ was incomplete and progressed to only $35 \mathrm{~cm}$ from the anal margin due to bad tolerance and a local inflammatory response related to severe diverticulosis.

CTC was requested after this incomplete OC. Our patient received a low residue diet for 48 hours and a 24hour clear liquid diet prior to the CTC. Laxatives were also administered to allow colonic evacuation, as well as a stool marker for fecal tagging 24 hours prior to the CTC. Our patient was positioned in the left decubitus position and air was manually insufflated through an intrarectal small gauge Foley catheter according to our

\section{Biomed Central}



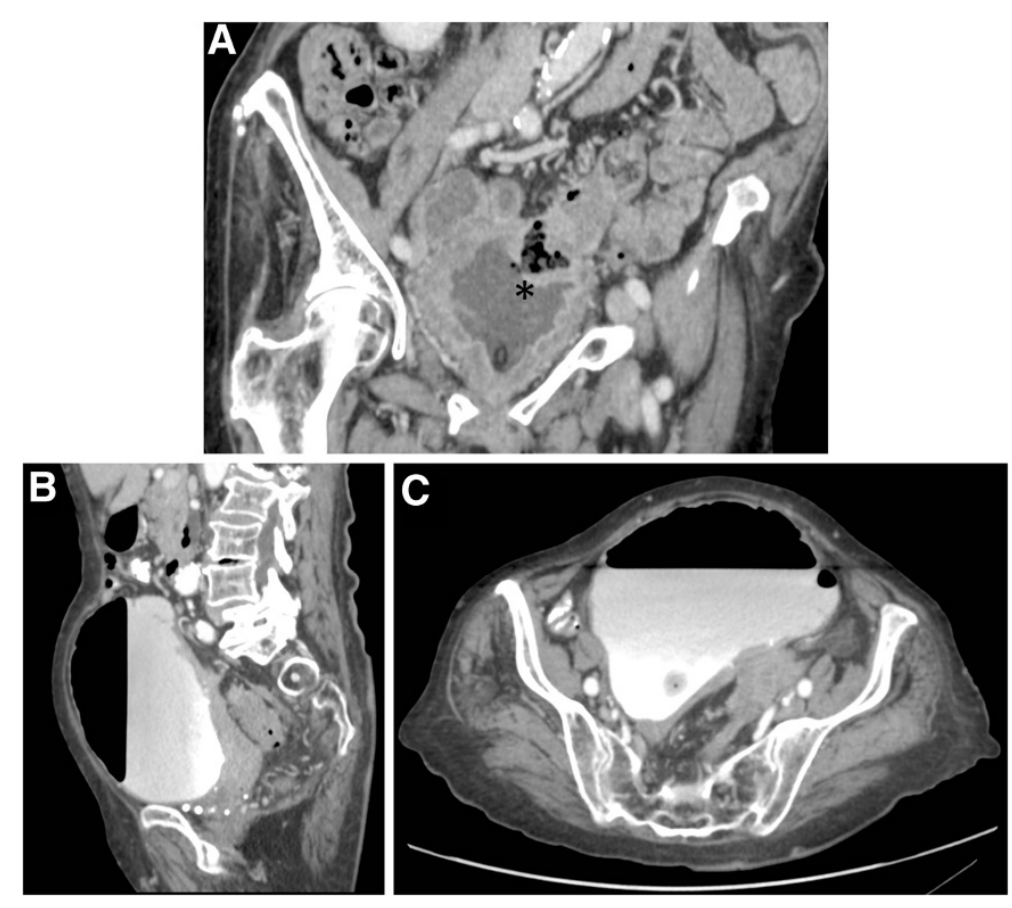

Figure 1 (A) Coronal reformatted CT acquired before the CTC shows an obvious colovesical fistula (asterisk). (B,C) After insertion of a Foley catheter and insufflation of air in the rectum, a great quantity of air appeared in the bladder, without colon distension.

patient's tolerance. Since the first scout image (with our patient in a supine position) revealed inadequate bowel distension, insufflations were continued and a second scout showed the presence of air in the pelvis but no colonic distension. Biphasic CT was performed, with our patient first in a prone position, then in a supine position. The second acquisition was realized after injection of $120 \mathrm{~mL}$ of iodinated contrast media (Accupaque 350, GE Healthcare, Glattbrugg, Switzerland). The CT parameters were as follows: $120 \mathrm{kV}, 120 \mathrm{~mA}$ (modulated) and $2 \mathrm{~mm}$ slice thickness with an interval of $1 \mathrm{~mm}$, corresponding to a dose length product of $206 \mathrm{mGy} \cdot \mathrm{cm}$ for the non-enhanced phase and $436 \mathrm{mGy} \cdot \mathrm{cm}$ for the contrastenhanced phase.

The images acquired showed a significant distension of her bladder, with an estimated volume of $1,350 \mathrm{~mL}$ and filled with contrast, and the presence of an intravesical air-fluid level. There was no colonic distension. The twodimensional multiplanar analysis of the images successfully revealed the fistulous tract between her bladder and her sigmoid colon but an analysis of her bowel lumen was not performed due to inadequate colonic distension (see Figure 1). Cystoscopy revealed a vesical opening with fecal material leakage $2 \mathrm{~cm}$ to $3 \mathrm{~cm}$ superior to the left ureterovesical junction, with no extrinsic visible lesion. Our patient underwent a sigmoidectomy with resection of the fistulous tract. Histopathology revealed a severe diverticulosis with focal diverticulitis of her sigmoid colon that had perforated into her bladder. Fibrotic tissue was present, but no neoplastic cells were identified.

\section{Discussion}

CTC is a robust imaging technique that is able to visualize the lumen of the colon and to detect the presence of polyps and masses. Thus, CTC could be a suitable alternative if $\mathrm{OC}$ is impossible or incomplete.

OC is not accepted by all patients and has several contraindications, such as severe comorbidity, bleeding disorders, advanced age, allergic reaction to anesthesia or sedation [1]. As many as $6 \%$ to $26 \%$ of examinations performed are incomplete and fail to reach the caecum [5]. The reasons for incomplete OC may be colonic redundancy, angulation and fixation of colonic loops, severe diverticulitis, adhesions and spasms as well as insufficient preparation. In these cases or if $\mathrm{OC}$ is not technically feasible, CTC in now increasingly performed.

In order to be successful, CTC requires adequate patient preparation and data acquisition, as well as imaging assessment including both two-dimensional multiplanar 
and virtual three-dimensional endoscopic images. Under optimal conditions, that is, a clean, dry and completely distended colon, without CT artefacts, CTC has similar detection rates for advanced neoplasia to OC [6]. Some authors have demonstrated that CTC is also feasible in a non-cathartic-prepared colon with a performance similar to $\mathrm{OC}$, at least in a population with a high prevalence of neoplasms (for neoplasms of at least $6 \mathrm{~mm}$ diameter) [7]. Adding CTC after incomplete OC allows clinicians to obtain complete examination of the colon in $91 \%$ of cases [5].

The majority of technical errors arise from residual stool and fluid, colic spasms and insufficient insufflations, as well as respiratory, stair steps and metallic artefacts [8]. When cleansing or distension of the colon is suboptimal, the rate of false positives or false negatives increases, leading to a decreased diagnostic performance of CTC.

Sufficient bowel distension is crucial for the accurate assessment of intraluminal colonic defects such as polyps. An underinflated bowel has luminal narrowing and some colonic segments are collapsed, leading to interpretation errors. Finally, CTC images are acquired in both supine and prone positions, in order to shift the position of fluid and retained feces and to visualize more accurately the nondependent mucosal-luminal interface of all colonic segments.

\section{Conclusion}

We report here a case of unsuccessful CTC due to insufficient colon distension related to a colovesical fistula. Colonic distension failed because all the insufflated air accumulated in the bladder. Clinicians have to be aware that a colovesical fistula may preclude successful CTC and thus may be a relative contraindication to CTC.

\section{Consent}

Written informed consent was obtained from the patient for publication of this case report and any accompanying images. A copy of the written consent is available for review by the Editor-in-Chief of this journal.

\section{Competing interests}

The authors declare that they have no competing interests.

\section{Authors' contributions}

$A N, R B$ and $X M$ were directly involved in the management of the patient. AN, RB and XM wrote the manuscript with support from DB and ST. CDB and $\mathrm{XM}$ reviewed and interpreted the $\mathrm{CT}$ colonography images. All authors read and approved the final manuscript.

Received: 5 October 2011 Accepted: 20 March 2012

Published: 23 July 2012

\section{References}

1. Blachar A, Sosna J: CT colonography (virtual colonoscopy): technique, indications and performance. Digestion 2007, 76:34-41.
2. Ing A, Lienert A, Frizelle F: Medical image. CT colonography for colovesical fistula. N Z Med J 2008, 121:105-108.

3. Murata A, Miyagi Y, Osoegawa T, Tanaka M, Nakamura K: Detection of retroperitoneal fistula of the colon by CT colonography. Indian J Gastroenterol 2008, 27:256.

4. Nadir I, Ozin Y, Kilic ZM, Oguz D, Ulker A, Arda K: Colovesical fistula as a complication of colonic diverticulosis: diagnosis with virtual colonoscopy. Turk J Gastroenterol 2011, 22:86-88.

5. Javeri K, Williams TR, Bonnett JW: An overview of the method, application, and various findings of computed tomographic colonography in patients after incomplete colonoscopy. Curr Probl Diagn Radiol 2010, 39:262-274.

6. Kim DH, Pickhardt PJ, Taylor AJ, Leung WK, Winter TC, Hinshaw JL, Gopal DV Reichelderfer M, Hsu RH, Pfau PR: CT colonography versus colonoscopy for the detection of advanced neoplasia. N Engl J Med 2007, 357:1403-1412.

7. Johnson CD, Manduca A, Fletcher JG, MacCarty RL, Carston MJ, Harmsen WS, Mandrekar JN: Noncathartic CT colonography with stool tagging: performance with and without electronic stool subtraction. AJR Am J Roentgenol 2008, 190:361-366.

8. Mang T, Maier A, Plank C, Mueller-Mang C, Herold C, Schima W: Pitfalls in multi-detector row CT colonography: a systematic approach. Radiographics 2007, 27:431-454.

doi:10.1186/1752-1947-6-214

Cite this article as: Neroladaki et al.: Colovesical fistula causing an uncommon reason for failure of computed tomography colonography: a case report. Journal of Medical Case Reports 2012 6:214.

\section{Submit your next manuscript to BioMed Central and take full advantage of:}

- Convenient online submission

- Thorough peer review

- No space constraints or color figure charges

- Immediate publication on acceptance

- Inclusion in PubMed, CAS, Scopus and Google Scholar

- Research which is freely available for redistribution 